\title{
Targeting the glycans of gp120: a novel approach aimed at the Achilles heel of HIV
}

\author{
Jan Balzarini
}

Lancet Infect Dis 2005;

5: 726-31

$J B$ is professor of virology and chemotherapy at the Rega Institute for Medical Research, Katholieke Universiteit Leuven,

Leuven, Belgium.

Correspondence to:

Professor Jan Balzarini, Rega Institute for Medical Research Katholieke Universiteit Leuven, Minderbroedersstraat 10 B-3000 Leuven, Belgium. Tel +3216337352 fax +3216337340 jan.balzarini@rega.kuleuven.be

The development of drug resistance in HIV compromises the long-term efficacy of current therapies. Furthermore, vaccine development faces huge problems, mainly because of the low antigenicity and immunogenicity of the HIV envelope glycoprotein gp120 and the efficient hiding of highly immunogenic epitopes by its glycans. There is evidence that mutant HIV strains containing glycosylation site deletions trigger the production of specific neutralising antibodies to previously hidden gp120 epitopes. I present a hypothesis that development of resistance against drugs that target the glycans on gp120 would result in a marked enhancement of neutralisation of HIV by the immune system-ie, drugs directed against the carbohydrate component of gp120 will select for mutant virus strains that progressively gain deletions in the glycosylation sites of gp120. Previously hidden epitopes would then be uncovered, and the virus will become highly susceptible to markedly increased immunological neutralisation. I believe this novel approach may become an entirely new therapeutic concept that exploits the high mutation rate of HIV and allows drug therapy to act in concert with a triggered immune response to suppress HIV more efficiently. Moreover, this approach could be applied to treat other chronic infections by viruses that contain a glycosylated envelope (eg, hepatitis B and C).

\section{Why does the immune system fail to efficiently control HIV infections?}

Antibodies against HIV produced by the humoral immune system bind both to free virus and virusinfected cells. ${ }^{1}$ The antibodies bind to the HIV envelope protein gp120, directly blocking virus infection (ie, neutralisation) or triggering effector systems that lead to viral clearance. Antiviral activity can be mediated by both neutralising and non-neutralising antibodies. Neutralising antibodies bind to viral proteins that are expressed on the envelope of the free virus particles, whereas non-neutralising antibodies bind to viral proteins mainly expressed on virus-infected cells but not substantially expressed on free virus particles. Generally, neutralising antibodies produced by the humoral immune system are crucial for vaccine-mediated protection against viral diseases. They may act by decreasing the viral efficiency of infection, which is then resolved by the cellular immune system. In fact, neutralisation occurs when a fairly large proportion of available sites on the virion are occupied by antibody, which leads to inhibition of virus attachment to host cells or to interference with the viral entry (fusion) process.

The immune system is not able to efficiently suppress HIV because gp120 does not elicit an efficient neutralising antibody response in infected people. ${ }^{2}$ This inefficient response is the result of several factors. First, the virus has low antigenicity, since little of the envelope surface of primary viruses is accessible for antibody binding, probably because of oligomerisation of gp120 proteins and the high degree of protein glycosylation (approximately 50\% of HIV-1 gp120 is glycan). Second, the virus has low immunogenicity-the mature carbohydrate oligomers constituting the envelope spikes of HIV appear to stimulate only weak antibody responses. Third, the virus has a high mutational rate; the resulting intensive viral variation compromises efficient neutralisation by the immune system. Wei and co-workers ${ }^{3}$ showed that gp120's glycan shield evolves during the course of infection in the face of a continuously changing antibody repertoire. Indeed, successive populations of escape virus in patients with acute HIV infection contained mutations in the env gene-which encodes gp120 - that were unexpectedly sparse and primarily involved changes in N-linked glycosylation sites. These continuous changes in glycan packing efficiently prevent neutralising antibody binding but not receptor binding. Thus, it could be hypothesised that the abundant glycosylation sites at the surface of the gp120 glycoprotein serve to protect against humoral immune responses to gp120 epitopes critical for HIV infectivity, transmission, or both. ${ }^{4}$ Indeed, carbohydrate regions of glycoproteins are considered as poor immunogens for several reasons. First, carbohydrate moieties exhibit microheterogeneity-one protein sequence can exhibit a broad range of glycoforms, causing the negation of any single antigenic response. ${ }^{5}$ Second, large carbohydrates are flexible and extend considerably from the protein core, and are thus able to cover potentially highly immunogenic epitopes. ${ }^{6}$ Finally, viruses depend on the host glycosylation machinery, and therefore, the glycans attached to viral proteins are quite similar to those attached to host glycoproteins, thus there is better tolerance of these carbohydrates?

Variants of HIV have been shown to exist in which peptide epitopes recognised by HIV-specific T cells have selected for exactly the type of mutation that converts these peptide ligands into antagonists, thus actively silencing HIV-specific T lymphocytes. ${ }^{8,9}$ Also viruses (including HIV) may try to evade the MHC class Imediated antigen presentation. ${ }^{10,11}$ Indeed, pathogens that attenuate MHC class I expression would have a 
selective advantage through elimination of class I molecules from the cell surface (ie, by downregulating transcription of class I genes through the action of the HIV protein $\mathrm{Nef}^{10}$ ). The infected cell then becomes temporarily invisible to cytotoxic $\mathrm{T}$ lymphocytes, giving the pathogen time to replicate. However, such a strategy is not without risk to the virus: natural killer cells can recognise cells deficient in self-MHC products. ${ }^{11}$

\section{Emergence of neutralising antibodies against gp120 with deleted glycosylation sites}

There is a wealth of evidence-both direct and indirect-that the glycan shield of HIV prevents the immune system from an efficient neutralising attack against the virus. Lee and colleagues ${ }^{12}$ identified five N-glycosylation sites on gp120 that, when deleted, resulted in compromised viral infectivity. Schonning and co-workers ${ }^{13}$ demonstrated that HIV-1 strains lacking the highly conserved N-linked glycan at position 306 within the V3 loop of gp120 are highly sensitive to neutralisation. Molecular clones of HIV lacking this Asn306 glycosylation site reacquired the site under invitro selection with monoclonal antibodies directed against the V3 loop. Bolmstedt and co-workers ${ }^{14}$ showed that glycosylation at Asn306 shields HIV-1 from neutralising antibodies. Importantly, Reiter and colleagues $^{15}$ convincingly demonstrated that rhesus monkeys infected with mutant simian immunodeficiency virus (SIV) strains lacking dual combinations of two N-linked glycosylation sites in the external envelope protein showed markedly increased antibody binding to specific peptides from this env region and also were substantially neutralised by the immune system. These results demonstrated that $\mathrm{N}$ glycosylation in SIV env has a role in limiting the neutralising antibody response to SIV and in shielding the virus from immune recognition. ${ }^{15}$ The results also illustrate that deletion of as few as two glycosylation sites in the viral env gene is sufficient to trigger a neutralising antibody response. Chackerian and colleagues ${ }^{16}$ and Cheng-Mayer and co-workers ${ }^{17}$ found that specific Nlinked glycosylation modifications in the envelope VI domain of SIV or in a SIV/HIV hybrid variant in vivo evolve in the host and alter recognition by neutralising antibodies. Finally, Kang and colleagues ${ }^{18}$ recently reported that modified HIV env-encoded proteins with reduced glycosylation in the domains surrounding the CD4 binding site or in the variable loop expose important neutralising epitopes at much higher levels than wild-type virus and may provide a tool for novel vaccine immunogens.

\section{Generation of glycosylated proteins}

The addition of carbohydrates to a dolicholphosphate carrier starts at the cytosolic surface of the endoplasmic reticulum membrane and continues at the luminal side of the endoplasmic reticulum membrane until the core oligosaccharide consisting of two N-acetylglucosamine, nine mannose, and three glucose residues $\left(\mathrm{GlcNAc}_{2} \mathrm{Man}_{9} \mathrm{Glc}_{3}\right)$ is completed and transferred to asparagine residues of a nascent growing polypeptide chain through the 1-position of $\mathrm{N}$-acetylglucosamine (figure 1). Potential glycosylation sites are indicated by the presence of the consensus sequence Asn-X-Ser/Thr within the nascent polypeptide. The three terminal glucose residues are then trimmed off by glucosidase I and II, and the terminal $\alpha-1,2$-mannose residue is removed by endoplasmic reticulum mannosidases. The newly glycosylated protein then moves to the Golgi complex, where further $\alpha$-1,3-mannose and $\alpha-1,6-$ mannose trimming occurs. Addition of one $\mathrm{N}$ acetylglucosamine residue is followed by the trimming of two more mannose residues. During the subsequent terminal glycosylation process, new terminal sugar residues-eg, N-acetylglucosamine, fucose, galactose, and sialic acid—can be added. Only one of the many possible terminal glycosylation pathways is shown in figure 1, and the number of branches generated is variable. The process results in a highly diverse array of oligosaccharides present on the peptide. ${ }^{19}$ The carbohydrates that are built on the two terminal mannose residues of the conserved pentasaccharide core can widely vary depending on the type of protein, cell, and species. However, gp120's glycans contain an unusually high amount of mannose residues, in particular $\alpha$-1,2-mannose oligomers at the surface of the glycan and $\alpha$-1,3-mannose and $\alpha$-1,6-mannose oligomers between the $\alpha-1,2$-mannose oligomer at the

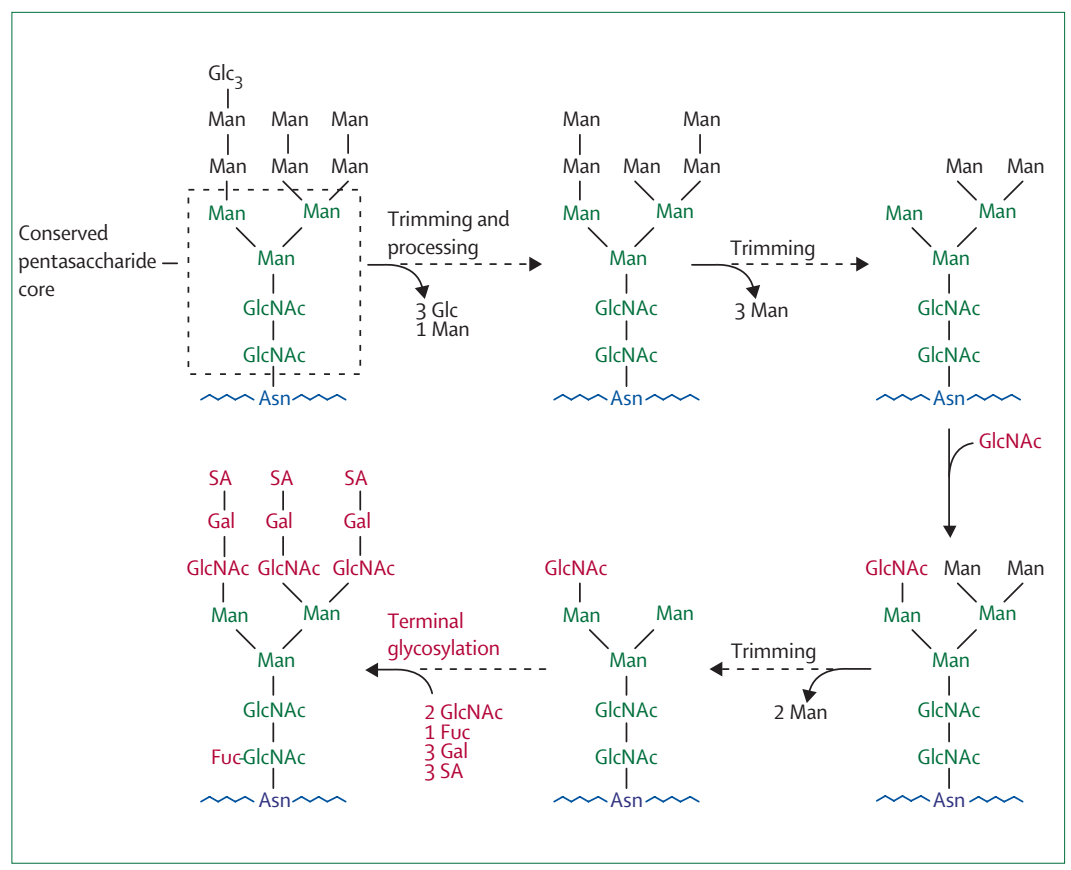

Figure 1: Biosynthesis of $\mathrm{N}$-linked core oligosaccharides

Asn=asparagine; Fuc=fucose; Gal=galactose; Glc=glucose; GlcNAc=N-acetylglucosamine; Man=mannose; $\mathrm{SA}=$ sialic acid. 


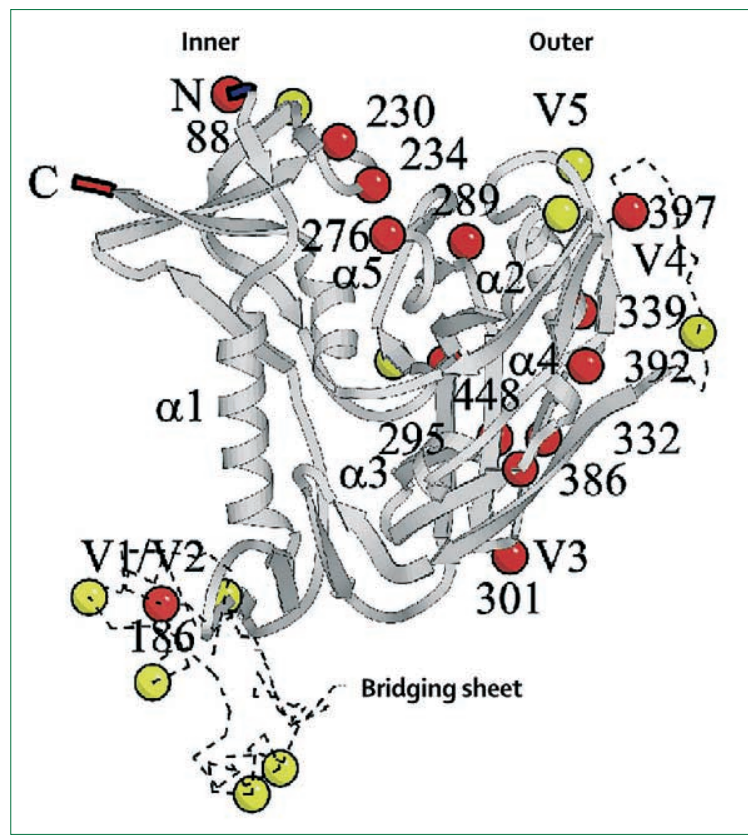

Figure 2: Structure of HIV-1 gp120 $0^{35}$

Glycosylation sites present in $\mathrm{HIV}-1(\mathrm{III}) \mathrm{gp} 120$ are indicated as balls. ${ }^{20}$ Those glycosylation sites that were deleted upon selection of HIV-1 in the presence of mannose-binding agents (GNA, HHA, and cyanovirin) $)^{33.34}$ are coloured in red. Two areas on the V1/V2 and V4 parts of gp120 are not resolved in the crystal structure. These areas are modelled by the broken lines. Figure courtesy of M Froeyen, Rega Institute, Leuven, Belgium.

surface of the glycan tree and the two Nacetylglucosamine residues linked to the protein. ${ }^{20}$

\section{Antiviral activity of carbohydrate-binding proteins}

A variety of carbohydrate-binding plant proteins known as agglutinins are endowed with anti-HIV activity. Most carbohydrate-binding plant proteins that show anti-HIV activity are specific for $\alpha-1-3-$ mannose and $\alpha-1-6$ mannose oligomer binding. ${ }^{21-24}$ Mannose-binding proteins have also been isolated and characterised from prokaryotic organisms-eg, cyanovirin from the greenblue algae Nostoc ellipsosporum ${ }^{25,26}$ and scytovirin from the cyanobacterium Scytonema varium. ${ }^{27}$ A striking exception is UDA, a plant protein derived from the stinging nettle Urtica dioica, ${ }^{22}$ which shows specificity for $\mathrm{N}$-acetylglucosamine. ${ }^{28,29}$ These agents have been shown to inhibit the entry process of the virus, in particular fusion. ${ }^{21}$ They not only inhibit HIV infection but also prevent HIV transmission by efficiently blocking cell-tocell contact. Therefore, these proteins have been suggested as potential microbicidal drugs. ${ }^{30}$ Cyanovirin has been shown to be efficacious in the prevention of virus infection in rhesus monkeys, providing proof-ofconcept. $^{31}$ It is thought that agglutinins exert their antiviral action by strongly binding to the sugar moieties present on gp120, thereby compromising the required conformational changes in gp120/gp41 for optimal interaction with the (co)-receptors and fusion with the target cell membrane.

\section{Deletion of $\mathrm{N}$-glycosylation sites in gp120 by carbohydrate-binding proteins}

A variety of entry inhibitors-eg, dextran sulfate and AMD3100 - select for virus strains that contain gp120 mutations. ${ }^{32}$ These mutations allow the virus to escape drug pressure in cell culture. Interestingly, the mannose-binding agglutinins were able to fully suppress the replication of these mutant virus strains..$^{33}$ Virus strains that emerged in the presence of escalating carbohydrate-binding protein concentrations predominantly showed mutations at N-glycosylation sites in gp120 but not gp41. ${ }^{33,34}$ The degree of resistance correlated well with the number of deletions of the $\mathrm{N}$ glycosylation sites in gp120. Monoclonal antibodies-eg, 2G12-for which the epitope is located in the area of aminoacid residues Asn295, Asn332, and Asn392 of gp120 35,36 lose antiviral activity once one or two of these $\mathrm{N}$-glycosylation sites were mutated. ${ }^{33,34}$ At the highest drug concentrations, virus isolates contained up to seven or eight different aminoacid mutations either at the $\mathrm{N}$ glycosylation sites or at the Ser/Thr aminoacid position in the conserved glycosylation consensus sequence. Figure 2 shows the putative glycosylation sites in HIV$1\left(\mathrm{III}_{\mathrm{B}}\right)$ gp120. The red-coloured balls represent those $\mathrm{N}$ glycosylation sites that are deleted upon exposure to the mannose-binding proteins Hippeastrum hybrid agglutinin (HHA), Galanthus nivalis agglutinin (GNA), and cyanovirin. ${ }^{33,34}$ Interestingly, these drug-resistant virus strains remain fully sensitive to other HIV entry inhibitors. ${ }^{33}$ Witvrouw and colleagues ${ }^{37}$ showed that cyanovirin selected-besides two point mutations at glycosylation sites 302 and 418-for a deletion in the V4 area of gp120 in which at least three high-mannose glycans were located, resulting in a virus strain with substantial resistance to this compound.

Thus, carbohydrate-binding proteins represent a unique class of conceptually novel anti-HIV compounds that select for an unusually specific and selective drugresistance profile. They represent the first molecules that predominantly select for deletions of glycosylation sites in HIV gp120. The mannose-binding GNA and HHA proved non-toxic against the proliferation of mammalian cells in cell culture, non-mitogenic (by contrast with phytohaemagglutinin), and not antimetabolically active. Intravenous bolus injection of $50-100 \mathrm{mg} / \mathrm{kg}$ in adult mice did not result in any visible side-effects. ${ }^{30}$ These observations indicate that certain agglutinins do not show substantial toxicity, and thus targeting mannose residues present on gp120 could become a rather safe approach to develop novel classes of non-toxic, carbohydrate-binding antiviral compounds. In fact, HIV$1\left(\right.$ III $\left._{\mathrm{B}}\right)$ gp120 contains about 24 potential N-glycosylation sites, 13 of which contain high complex mannose-type glycans, and 11 of which contain high-mannose or 
hybrid-type glycans. Glycoproteins with high mannosetype glycans are common in prokaryotes and viruses such as HIV, but are rather rare in mammalian cells. This distribution may be one of the main reasons why several mannose-specific agents show poor-if anytoxicity in mammalian cell models under experimental conditions where they fully inhibit virus entry. These observations are also in line with findings that cyanovirin proved effective in preventing SIV transmission in macaques in the absence of toxic sideeffects. ${ }^{31}$

\section{The carbohydrate-binding agent concept}

I believe that the use of carbohydrate-binding agents represents a novel therapeutic approach of HIV treatment that is entirely different in concept from all currently existing therapeutic methods. Exposure of HIV to carbohydrate-binding agents puts the virus in a dilemma of either eventually becoming eliminated from its host by being kept suppressed by the carbohydratebinding agent, or escaping the pressure from the carbohydrate-binding agent by mutating (deleting) glycosylation sites in gp120, thereby becoming prone to neutralisation and elimination by the immune system (figure 3).

If this hypothesis is correct, it is conceivable that the concerted action of drug treatment and immune surveillance may markedly compromise the viability and infectivity of the virus. Whereas so far major attempts have been made to design and develop drugs that have as high a genetic barrier as possible to delay the development of drug resistance, the proposed approach makes use of viral variability and the inherent errorprone virus replication to generate mutant virus strains with deletions of gp120 glycosylation sites. Furthermore, the proposed approach makes use of a thus far unique concerted action of drug chemotherapy-triggering the immune system and combining chemotherapy and "therapeutic self-vaccination" in the host by the administration of one single drug. Moreover, one could even consider vaccination with partly deglycosylated gp120 before, or at the start of, therapy with carbohydrate-binding agents. Exposure of HIV to such agents will hit the Achilles heel of the virus-ie, the hiding of its highly immunogenic and antigenic epitopes on gp120 by keeping a dense glycan shield. Taking this life-saving strategy away, the virus will become prone to continuous neutralisation by the immune system. Transmission of HIV predominantly occurs through binding of gp120 with the glycanbinding DC-SIGN, after which the virus is exposed to T lymphocytes by DC-SIGN-expressing dendritic cells. Thus, it is conceivable that the efficient binding of DCSIGN to HIV with mutated gp120 will become suboptimal. In addition, since glycosylation of the native envelope protein helps both to correctly fold gp160 and to subsequently convert to gp120 and gp41, it is expected that an increasing amount of deleted glycosylation sites in the precursor gp160 molecule will affect these processes, resulting in compromised virulence/ infectivity.

These considerations are based on the assumption that carbohydrate-binding agents will predominantly select for gp120 glycosylation mutants in the natural host, as they do in cell culture, and that gp120 would not evolve via alternative mechanisms in response to pressure from these agents. Furthermore, one can not exclude the possibility that selected gp120 mutations may allow the envelope glycoprotein to become predominantly glycosylated by complex-type, rather than highmannose-type carbohydrates and may attempt to escape anti-mannose drug mediated neutralisation. Indeed, it is thought that an abundant presence of complex carbohydrates on gp120 can prevent antibody binding equally well as high-mannose type glycans. Although this mechanism has to be proven to be used in vivo by the virus in an attempt to escape drug pressure (it clearly does not in vitro ${ }^{33,34}$ ), it should be kept in mind that $\alpha-1,3$ mannose and $\alpha$-1,6-mannose residues are still invariably present in each of the complex-type glycans, and that carbohydrate-binding agents targeting other sugar specificities (eg, N-acetylglucosamine, sialic acid, galactose, fucose, etc) should also be considered as potentially valuable tools.

\section{Evidence from in-vivo studies}

At least two in-vivo studies have been carried out that suggest that the use of carbohydrate-binding agents may be realistic, viable, and achievable. Igarashi and coworkers ${ }^{38}$ demonstrated that rhesus macaques receiving a continuous infusion of cell-free HIV-1 particles showed a considerably lower virion half-life, and the

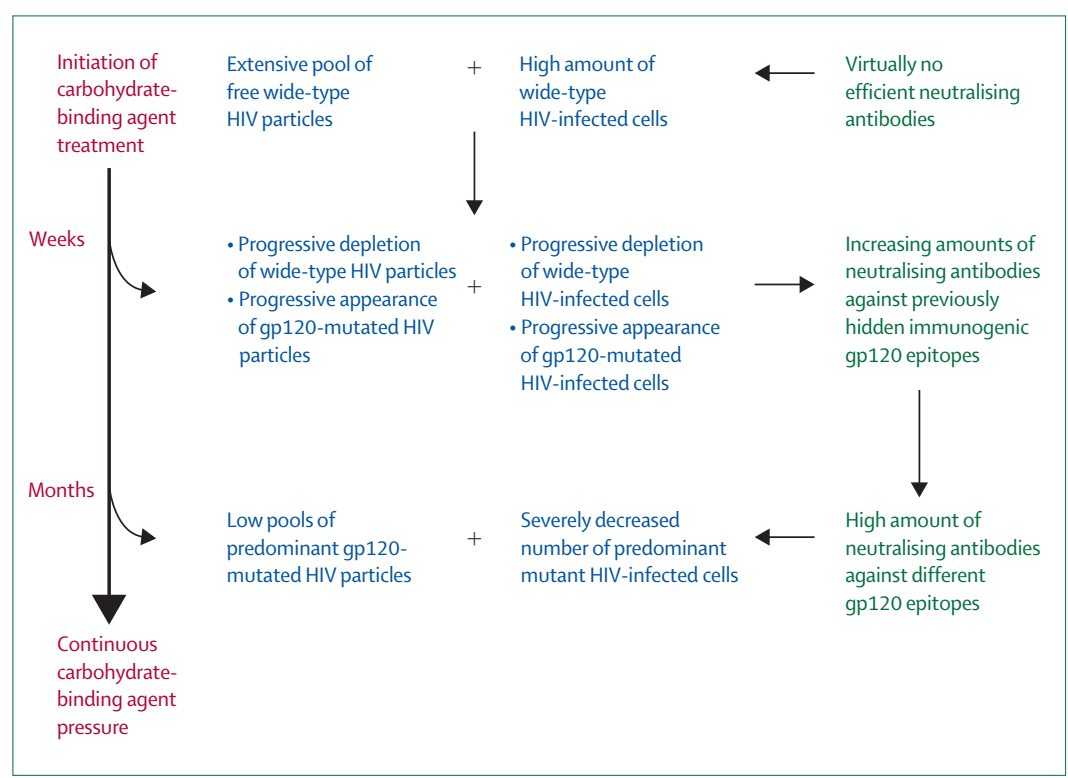

Figure 3: Schematic overview of the carbohydrate-binding agent concept 
virus eventually became undetectable in blood if the monkeys had high-titre HIV-1-specific neutralising antibodies, compared with those animals that lacked virus-specific neutralising antibodies. Moreover, Reiter and colleagues ${ }^{15}$ showed that monkeys exposed to SIV strains that contain two deleted glycosylation sites in the env gene produced a high-titre of neutralising antibodies and exhibited a dramatic drop in infectious virus titre in the plasma compared with wild-type virus. Thus, both studies provide strong evidence that administration of carbohydrate-binding agents to virus-infected animals may trigger the immune reaction and cause a drop in viral load.

\section{Testing the hypothesis}

The hypothesis could most likely be validated by selecting SIV strains in cell culture in the presence of carbohydrate-binding agents and then infecting animals with either the parent wild-type or mutant SIV strains in the presence or absence of the drug. A more direct proofof-concept could be obtained by simply treating SIVinfected animals with carbohydrate-binding agents and monitoring the (neutralising) antibody response, the specificity of the generated antibodies, and the titre of SIV in plasma and relevant compartments or organs of the exposed animals. Furthermore, the use of carbohydrate-binding agents should not only be effective against HIV, but also against chronic infections with other viruses containing a glycosylated envelope-eg, hepatitis $\mathrm{B}$ and $\mathrm{C}$ viruses. A marked number of HIVinfected individuals are coinfected by hepatitis $\mathrm{B}$ or hepatitis $\mathrm{C}$ virus; one single carbohydrate-binding agent could be effective against both viruses at the same time.

Although carbohydrate-binding proteins may not be very convenient to be used as systemic therapeutic agents, compounds of low molecular weight that bind to carbohydrates would be much more convenient. In fact, the antibiotic pradimicin A and several of its derivatives (eg, benanomicin), originally found in the fermentation broth of Actinomadura hibisca, ${ }^{39,40}$ bind to D-mannosides. Moreover, pradimicin A has been shown to be possess antifungal activity ${ }^{41,42}$ and also be able to inhibit HIV infection of $\mathrm{T}$ cells through the interaction with the high mannose-type oligosaccharides on gp $120 .{ }^{43}$ Therefore, we believe that pradimicin A should be considered as a low molecular weight carbohydrate-binding agent prototype that might be helpful to prove the concept in vivo.

\section{Novelty and uniqueness of the carbohydrate- binding agent approach}

The proposed hypothesis differs from existing treatment methods by at least seven important characteristics. First, the appearance of mutations should be avoided with current therapeutic drugs for HIV; however, they may have potential benefit when using carbohydrate-binding agents. Second, whereas drug resistance development weakens or annihilates the efficacy of existing drugs, the appearance of resistant viruses would increasingly potentiate the (immuno)therapeutic action of carbohydrate-binding agents. Third, while all existing anti-HIV drugs interact with their target protein in a stoichiometric manner (ie, one drug molecule binds to one target protein molecule), many carbohydrate-binding agents interact with a target gp120 molecule at the same time-there are 20-29 glycans on one single gp120 molecule, and many gp120 molecules on a single virus particle or virus-infected cell. This high level of binding results in a high genetic barrier for HIV to overcome. Fourth, current highly active antiretroviral therapy (HAART) consists of a combination of three or more compounds administered at the highest possible dose; by contrast, the carbohydrate-binding agents may (perhaps preferentially) be given as monotherapy before HAART comes into the picture. Fifth, none of the existing antiHIV drugs (perhaps with the exception of lamivudine) directly or indirectly interact with the immune system to exert their antiviral potential. By contrast, carbohydratebinding agents will likely trigger a strong immune response by initiating production of neutralising antibodies, in addition to their direct, purely antiviral effect. Sixth, the deletion of glycosylation sites in gp120 may not only trigger production of neutralising antibodies against previously hidden strong immunogenic epitopes of gp120, but will likely delay the initial spread of virus from DC-SIGN-containing dendritic cells to $T$ lymphocytes also. However, it should be expected that HIV with glycosylation site deletions will revert by reincorporating the sites when transmitted to another individual who is not receiving carbohydrate-binding agent treatment. Seventh, and finally, carbohydratebinding agent treatment may result in attenuated virus strains with lower virulence (infectivity) due to compromised folding and conversion of gp160 to gp120 and gp41. Thus, carbohydrate-binding agent exposure will concomitantly have multiple effects on different aspects of virus infection. I therefore believe that an intensive search for low molecular weight carbohydrate-binding agents should start right away, and further investigation of this principle be undertaken as soon as possible.

\section{Conflicts of interest}

I declare that I have no conflicts of interest.

\section{Acknowledgments}

JB is supported by grants from the European Commission (René Descartes Prize-2001 number HPAW-2002-900001 and EMPRO number 503558 of the 6th Framework Programme), the Agence Nationale de Recherche sur le SIDA (France), the Fonds voor Wetenschappelijk Onderzoek - Vlaanderen (FWO) Krediet G-0267-04, and the Centers of Excellence of the Katholieke Universiteit Leuven (number EF/05/15).

References

1 Burton DR. Antibodies, viruses and vaccines. Nature Rev Immunol 2002; 2: 706-13.

2 Burton DR. A vaccine for HIV type 1: the antibody perspective. Proc Natl Acad Sci USA 1997; 94: 10018-23.

3 Wei X, Decker JM, Wang S, et al. Antibody neutralization and escape by HIV-1. Nature 2003; 422: 307-12. 
4 Rudd PM, Dwek RA. Glycosylation: heterogeneity and the 3D structure of proteins. Crit Rev Biochem Mol Biol 1997; 32: 1-100.

5 Rudd PM, Elliott T, Cresswell P, Wilson IA, Dwek RA Glycosylation and the immune system. Science 2001; 291: 2370-75.

6 Wilson IA, Stanfield RL. A Trojan horse with a sweet tooth. Nat Struct Biol 1995; 2: 433-36.

7 Scanlan CN, Pantophlet R, Wormald MR, et al. The broadly neutralizing anti-human immunodeficiency virus type 1 antibody 2 G12 recognizes a cluster of alpha1 $\rightarrow 2$ mannose residues on the outer face of gp120. J Virol 2002; 76: 7306-21.

8 Bertoletti A, Sette A, Chisari FV, et al. Natural variants of cytotoxic epitopes are T-cell receptor antagonists for antiviral cytotoxic T cells. Nature 1994; 369: 407-10.

9 Klenerman P, Rowland-Jones S, McAdam S, et al. Cytotoxic T-cell activity antagonized by naturally occurring HIV-1 Gag variants. Nature 1994; 369: 403-07.

10 Schwartz O, Marechal V, Le Gall S, Lemonnier F, Heard JM. Endocytosis of major histocompatibility complex class I molecules is induced by the HIV-1 Nef protein. Nat Med 1996; 2: 338-42.

11 Ploegh HL. Viral strategies of immune evasion. Science 1998; 280: 248-53.

12 Lee WR, Syu WJ, Du B, et al. Nonrandom distribution of gp120 Nlinked glycosylation sites important for infectivity of human immunodeficiency virus type 1. Proc Natl Acad Sci USA 1992; 89: 2213-17.

13 Schonning K, Jansson B, Olofsson S, Hansen JES. Rapid selection for an N-linked oligosaccharide by monoclonal antibodies directed against the V3 loop of human immunodeficiency virus type 1 . J Gen Virol 1996; 77: 753-58.

14 Bolmstedt A, Hinkula J, Rowcliffe E, Biller M, Wahren B, Olofsson S. Enhanced immunogenicity of a human immunodeficiency virus type 1 env DNA vaccine by manipulating $\mathrm{N}$-glycosylation signals. Effects of elimination of the V3 306 glycan. Vaccine 2001; 20: 397-05.

15 Reiter JN, Means RE, Desrosiers RC. A role for carbohydrates in immune evasion in AIDS. Nat Med 1998; 4: 679-84.

16 Chackerian B, Rundensey LM, Overbaugh J. Specific N-linked and O-linked glycosylation modifications in the envelope VI domain of simian immunodeficiency virus variants that evolve in the host alter recognition by neutralizing antibodies. J Virol 1997; 71 7719-27.

17 Cheng-Mayer C, Brown A, Harouse J, Luciw PA, Mayer AJ. Selection for neutralization resistance of the simian human immunodeficiency virus SHIVSF33A variant in vivo by virtue of sequence changes in the extracellular envelope glycoprotein that modify N-linked glycosylation. J Virol 1999; 73: 5294-300.

18 Kang SM, Quan FS, Huang C, et al. Modified HIV envelope proteins with enhanced binding to neutralizing monoclonal antibodies. Virology 2005; 331: 20-32.

19 Helenius A, Aebi M. Intracellular functions of N-linked glycans. Science 2001; 291: 2364-69.

20 Leonard CK, Spellman MW, Riddle L, Harris RJ, Thomas JN, Gregory TI. Assignment of intrachain disulfide bonds and characterization of potential glycosylation sites of the type 1 recombinant human immunodeficiency virus envelope glycoprotein (gp120) expressed in Chinese hamster ovary cells. J Biol Chem 1990; 265: 10373-82.

21 Balzarini J, Schols D, Neyts J, Van Damme E, Peumans W, De Clerca E. $\alpha$-(1-3)- and $\alpha$-(1-6)-D-mannose-specific plant lectins are markedly inhibitory to human immunodeficiency virus and cytomegalovirus infections in vitro. Antimicrob Agents Chemother 1991; 35: 410-16.

22 Balzarini J, Neyts J, Schols D, et al. The mannose-specific plant lectins from Cymbidium hybrid and Epipactis helleborine and the $(\mathrm{N}$ acetylglucosamine) ${ }_{n}$-specific plant lectin from Urtica dioica are potent and selective inhibitors of human immunodeficiency virus and cytomegalovirus replication in vitro. Antiviral Res 1992; 18 191-207.
23 Van Damme EJM, Peumans WJ, Pusztai A, Barocz S, eds Handbook of plant lectins: properties and biomedical applications. Chichester, NY: John Wiley \& Sons, 1998.

24 Sharon N, Lis H, eds. Lectins, 2nd edn. Dordrecht: Kluwer Academic Publishers, 2003.

25 Boyd MR, Gustafson KR, McMahon JB, et al. Discovery of cyanovirin-N, a novel human immunodeficiency virus-inactivating protein that binds viral surface envelope glycoprotein gp120: potential applications to microbicide development. Antimicrob Agents Chemother 1997; 41: 1521-30.

26 Bolmstedt AJ, O'Keefe BR, Shenoy SR, McMahon JB, Boyd MR. Cyanovirin- $\mathrm{N}$ defines a new class of antiviral agent targeting $\mathrm{N}$ linked, high-mannose glycans in an oligosaccharide-specific manner. Mol Pharmacol 2001; 59: 949-54.

27 Bokesch HR, O'Keefe BR, McKee TC, et al. A potent novel antiHIV protein from the cultured cyanobacterium Scytonema varium. Biochemistry 2003; 42: 2578-84.

28 Shibuya N, Goldstein IJ, Shafer JA, Peumans WJ, Broekaert WF. Carbohydrate binding properties of the stinging nettle (Urtica dioica) rhizome lectin. Arch Biochem Biophys 1986; 249: 215-24.

29 Harata K, Muraki M. Crystal structures of Urtica dioica agglutinin and its complex with tri-N-acetylchitotriose. J Mol Biol 2000; 297: 673-81.

30 Balzarini J, Hatse S, Vermeire K, et al. Mannose-specific plant lectins from the Amaryllidaceae family qualify as efficient microbicides for prevention of human immunodeficiency virus infection. Antimicrob Agents Chemother 2004; 48: 3858-70.

31 Tsai CC, Emau P, Jiang Y, et al. Cyanovirin-N gel as a topical microbicide prevents rectal transmission of SHIV89.6P in macaques. AIDS Res Human Retrovir 2003; 19: 535-41.

32 Esté JA. HIV resistance to entry inhibitors. AIDS Rev 2001; 3: 121-32.

33 Balzarini J, Van Laethem K, Hatse S, et al. Profile of resistance of human immunodeficiency virus to mannose-specific plant lectins. J Virol 2004; 78: 10617-27.

34 Balzarini J, Van Laethem K, Hatse S, et al. Marked depletion of glycosylation sites in HIV-1 gp120 under drug pressure by the mannose-specific plant lectins of Hippeastrum hybrid and Galanthus nivalis. Mol Pharmacol 2005; 67: 1556-65.

35 Kwong PD, Wyatt R, Robinson J, Sweet RW, Sodroski J, Hendrickson WA. Structure of an HIV gp120 envelope glycoprotein in complex with the CD4 receptor and a neutralizing human antibody. Nature 1998; 393: 648-59.

36 Calarese DA, Scanlan CN, Zwick MB, et al. Antibody domain exchange is an immunological solution to carbohydrate cluster recognition. Science 2003; 300: 2065-71.

37 Witvrouw M, Fikkert V, Hantson A, et al. Resistance of human immunodeficiency virus type 1 to the high-mannose binding agents cyanovirin N and concanavalin A.J Virol 2005; 79: 7777-84.

38 Igarashi T, Brown C, Azadegan A, et al. Human immunodeficiency virus type 1 neutralizing antibodies accelerate clearance of cell-free virions from blood plasma. Nature Med 1999; 5: 211-16.

39 Oki T, Konishi M, Tomatsu K, et al. Pradimicin, a novel class of potent antifungal antibiotics. J Antibiot 1988; 41: 1701-04.

40 Gomi S, Sezaki M, Kondo S, Hara T, Naganawa H, Takeuchi T. The structures of new antifungal antibiotics, benenomicin A and B. $J$ Antibiot 1988; 41: 1019-28.

41 Oki T, Tenmyo O, Hirano M, Tomatsu K, Kamei H. Pradimicins A $B$ and $C$ : new antifungal antibiotics. II. In vitro and in vivo biological activities. J Antibiot 1990; 43: 763-70.

42 Kakushima M, Masuyoshi S, Hirano M, et al. In vitro and in vivo antifungal activities of BMY-28864, a water-soluble pradimicin derivative. Antimicrob Agents Chemother 1991; 35: 2185-90.

43 Tanabe-Tochikura A, Tochikura T, Yoshida O, Oki T, Yamamoto N. Pradimicin A inhibition of human immunodeficiency virus: attenuation by mannan. Virology 1990; 176: 467-73. 